Pacific Journal of Mathematics

STRUCTURES UNIFORMES FAIBLES SUR UNE CLASSE DE
CÔNES ET D'ENSEMBLS CONVEEXS 


\title{
STRUCTURES UNIFORMES FAIBLES SUR UNE CLASSE DE CÔNES ET D'ENSEMBLES CONVEXES
}

\author{
HICHAM FAKHOURY
}

\begin{abstract}
On donne des conditions nécessaires et suffisantes pour qu'un cone saillant $C$ recouvert par une famille dénombrable et filtrante croissante de convexes compacts $\left(K_{n}\right)_{n \in I N}$ soit complet pour la plus fine des structures uniformes faibles compatibles avec la topologie des compacts $\left(K_{n}\right)_{n \in N}$. Il en est ainsi lorsque pour tout entier $n$, le convexe est un chapeau de $C$. On en déduit que tout convexe fermé recouvert par une famille analogue de chapeaux peut etre muni d'une structure uniforme faiblement complète. Dans la dernière partie on compare diverses structures uniformes faibles sur le cone $C$, ce qui permet de donner une condition nécessaire et suffisante pour que la topologie induite par la plus fine des structures uniformes faibles compatibles avec la topologie des convexes compacts coincide avec la topologie de $C$.
\end{abstract}

I. Représentatiôn d'un cone convexe. Soient $E$ un espace localement convexe séparé (e.l.c.) et $C$ un cône convexe saillant qui engendre $E$. On appelle chapeau de $C$ tout convexe compact $K \subset C$ dont le complémentaire est convexe; un tel chapeau est universel s'il engendre $C$. Un cône est bien coiffé s'il est réunion de ses chapeaux. Dans la suite, on considérera la famille des convexes compacts de $C$; on peut donc, sans diminuer la généralité, supposer $E$ muni de la topologie affaiblie $\sigma\left(E, E^{\prime}\right)$. Si $F$ est un espace vectoriel en dualité séparante avec $E$ on notera $\sigma(C, F)$ la structure uniforme induite sur $C$ par $\sigma(E, F)$.

Soit $\left(K_{i}\right)_{i \in I}$ une famille de convexes compacts admettant l'origine comme point extrémal et recouvrant $C$; on note $A$ l'espace des formes linéaires sur $E$ dont la restriction à chaque convexe $K_{i}$ est continue. Cet espace sera muni de la topologie de la convergence uniforme sur tout convexe $K_{i}$. L'espace $A$ dépend en général de la famille $\left(K_{i}\right)_{i \in I}$ considérée (voir toutefois le corollaire 11). Si $\left.f\right|_{K_{i}}$ désigne la restriction de $f$ à $K_{i}$, il existe un système fondamental de voisinage de 0 dans $A$ formé par les intersections finies des ensembles:

$$
V_{i \varepsilon}=\left\{f \in A ;\left\|\left.f\right|_{K_{i}}\right\| \leqslant \varepsilon\right\} \text {. }
$$

On supposera l'espace $A$ ordonné par le cône $A_{+}$des fonctions de $A$ positives sur $C$. Ce cône est saillant car $C$ engendre $E$. Le dual $A^{\prime}$ de l'espace $A$ sera toujours muni de la topologie faible $\sigma\left(A^{\prime}, A\right)$ et de l'ordre dual de celui de $A$, son cône positif sera noté $A_{+}^{\prime}$. 
Proposition 1. L'espace E s'identifie (algébriquement) au dual $A^{\prime}$ de l'espace $A$ et $C$ à un sous-cône dense dans $A_{+}^{\prime}$. L'injection canonique de $C$ dans $A_{+}^{\prime}$ est continue sur chaque compact $K_{i}$, et $\sigma(C, A)$ est la plus fine des structures uniformes faibles compatibles avec la topologie des convexes $\left(K_{i}\right)_{i \in I}$.

DÉmonstration. Soit $E^{\prime}$ le dual de $E$; munissons $E^{\prime}$ de la topologie $T_{c}$ de la convergence uniforme sur tout convexe compact $K_{i}$. Les deux topologies $T_{c}$ et $\sigma\left(E^{\prime}, E\right)$ sont compatibles. En effet, $T_{c}$ peut s'exprimer comme la topologie de la convergence uniforme sur les ensembles $\left(c\left(K_{i} \cup-K_{i}\right)\right)_{i \in I}$. Or ces ensembles forment un recouvrement de $E$ par des parties convexes équilibrées et la propriété résulte du théorème de Mackey. D'autre part, l'espace $E^{\prime}$ est dense dans $A$. En effet, soient $f$ une fonction de $A$ et $\varepsilon>0$; pour toute partie finie $i_{1}, \cdots, i_{n}$ de $I$, il existe (d'après le théorème de Hahn-Banach) une fonction $h_{i_{1} \cdots i_{n}}, \varepsilon$ de $E^{\prime}$ telle que $\left|f-h_{i_{1} \cdots i_{n}}, \varepsilon\right|$ soit majorée par $\varepsilon$ sur le convexe compact $c\left(\cup_{r=1}^{n} K_{i r}\right)$. Ceci détermine une famille filtrante de fonctions de $E^{\prime}$ qui converge uniformément sur tout $K_{i}$ vers la fonction $f$. Par conséquent, le dual de $A$ s'identifie à $E$. L'injection canoique $j$ de $E$ sur $A^{\prime}$ s'écrit:

$j(x)(f)=f(x)$ pour tout $x$ dans $E$ et $f$ dans $A$.

Il est clair que $j(C) \subset A_{+}^{\prime}$.

La densité de $j(C)$ dans $A_{+}^{\prime}$ est une conséquence du théorème des bipolaires puisque le polaire de $C$ dans $A$ est identique à $A_{+}$. La continuité de $j$ sur tout convexe $K_{i}$ ainsi que la dernière assertion sont évidentes.

Les cônes $C$ et $A_{+}^{\prime}$ ne sont pas, en général, égaux; en effet, le cône de $\boldsymbol{R}^{2}$ engendré par le disque

$$
K=\left\{(x, y) \in R^{2} ; x^{2}+(y-1)^{2} \leqslant 1\right\}
$$

n'est pas fermé dans $\boldsymbol{R}^{2}$. Cependant, il y a égalité lorsque le cône $C$ est $\sigma\left(E, E^{\prime}\right)$ - fermé, donc à fortiori $\sigma(E, A)$-fermé. Dans la suite, nous donnerons d'autres conditions suffisantes pour que $C$ soit égal à $A_{+}^{\prime}$ dans le cas où la famille $\left(K_{i}\right)_{i \in I}$ est dénombrable.

II. Structures uniformes sur un cône $\boldsymbol{K}_{\sigma}$. Dans cette section nous supposons la famille $\left(K_{i}\right)_{i \in I}$ dénombrable. Nous commençons par éliminer les deux cas particuliers suivant: (a) Si le cône $C$ est engendré par un convexe compact $K$ ne contenant pas l'origine, $C$ est localement compact et faiblement complet. (b) Si le cône $C$ est réunion dénombrable d'une famille de convexes compacts et si de plus c'est un espace de Baire, $C$ est un cône localement compact et faiblement complet. 
Proposition 2. Soient $C$ le cône engendré par un convexe compact $K$ contenant l'origine, et $A$ l'espace des formes linéaires sur $E$ continues sur $K$. Si $C$ est $\sigma(C, A)$-complet le sous-espace $V=C \cap-C$ est de dimension finie.

Demonstration. Le cône $C$ est $\sigma(E, A)$-complet donc $\sigma(E, A)$ fermé, par suite $V=C \cap-C$ est fermé dans $C$ et $\sigma(C, A)$-complet. Or la topologie induite par $\sigma(E, A)$ sur $V$ est $\sigma\left(V, A / V^{\circ}\right)$-où $V^{\circ}$ désigne le polaire de $V$ dans $A$ - D'autre part $E=A^{\prime}$, par conséquent $V$ s'identifie à $\left(A / V^{\circ}\right)^{\prime}$ puisque $V=V^{\circ \circ}$. Comme $A / V^{\circ}$ est un Banach, $V$ ne peut être $\sigma\left(V, A / V^{\circ}\right)$-complet que si $A / V^{\circ}$ est de dimension finie. Il en est alors de même pour $V$.

Dans la suite, le cône $C$ sera toujours supposé saillant. Pour démontrer le théorème principal de cette partie, nous avons besoin des deux résultats suivants:

LeMme 3. Soient $F$ un e.l.c. et $X$ un cône convexe de sommet 0 vérifiant les deux conditions suivantes:

(a) $X$ est complet pour la structure uniforme induite par $F$.

(b) Le sommet 0 admet un système fondamental dénombrable de voisinages dans $X$.

Alors, les ensembles $V=U \cap X-U \cap X$ (où $U$ parcourt le filtre des voisinages de 0 dans $X$ ) forment une base de voisinages de 0 pour une topologie d'e.l.c. sur $H=X-X$, complète, métrisable et plus fine que celle induite par $F$.

Corollaire 4. Soit $F$ un espace de Frechet ordonné par un cône convexe fermé $X$ tel que $F=X-X$, toute forme linéaire positive sur $X$ est continue.

Le Corollaire 4 est une conséquance du Lemme 3 et du théorème des homomorphismes de Banach. Le Lemme 3 est démontré dans (7).

Dans la suite de cette section, nous supposerons l'espace $E$ ordonné par le cône $C$. Si $B$ est une partie de $C$ nous notons $\hat{B}$ le saturé de $B$, c'est-à-dire:

$$
\hat{B}=\{y \in C \text { tel qu'il existe } x \in B \text { avec } y \leqslant x\} .
$$

Rappelons que nous avons l'galiété $K=\hat{K}$ quand $K$ est un chapeau de $C$. Le théorème suivant étend des résultats antérieurs (6) et fournit une réciproque partielle.

THÉORÈme 5. Si le cône $C$ est recouvert par une famille dénombrable et filtrante croissante de convexes compacts, les propriétés 
suivantes sont équivalentes:

(a) $C$ est $\sigma(C, A)$-complet.

(b) Pour tout entier $n$, le convexe $\hat{K}_{n}$ est borné et complet pour $\sigma(C, A)$.

(c) Pour tout entier $n$, le convexe $\hat{K}_{n}$ est $\sigma(C, A)$-compact.

Démonstration. (a) $\Rightarrow$ (b) Le cône $C$ étant complet il est $\sigma(C$, $A)$ - fermé et il suffit de montrer que $\hat{K}_{n}$ est fermé dans $C$. Soient $y$ un point adhérent à $\hat{K}_{n}$, et $\left(y_{u}\right)_{u \in \mathcal{\nu}}$ un ultrafiltre porté par $\hat{K}_{n}$ convergent vers $y$; pour tout $u$ il existe $x_{u} \in K_{n}$ tel que $y_{u} \leqslant x_{u}$.

L'ultrafiltre $\left(x_{u}\right)_{u \in \mathscr{Y}}$ converge vers un point $x$ de $K_{n}$ et le cône $C$ étant $\sigma(C, A)$-fermé on a $y \leqslant x$; par suite $y \in \widehat{K}_{n}$. Pour montrer que $\hat{K}_{n}$ est $\sigma(C, A)$-borné remarquons que $A$ est positivement engendré (3); d'autre part si $f \in A_{+}$et $y \in \hat{K}_{n}$ il existe $x \in \hat{K}_{n}$ tel que $y \leqslant x$ et par conséquent $0 \leqslant f(y) \leqslant f(x) \leqslant\left\|\left.f\right|_{K_{n}}\right\|$.

(b) $\Rightarrow$ (c) est une conséquence d'une propriété générale des ensembles bornés faiblement complets.

(c) $\Rightarrow$ (a) Remarquons que l'espace $A$ est identique à l'espace des formes linéaires sur $E$ dont la restriction à chaque $\hat{K}_{n}$ est continue. L'espace $A$ muni de la topologie de la convergence uniforme sur tout $\left(\hat{K}_{n}\right)_{n \in N}$ est un espace de Frechet; d'après le théorème des homomorphismes de Banach, cette topologie est identique à celle de la convergence sur tout $\left(K_{n}\right)_{n \in N}$. Nous noterons $V_{n, \varepsilon}$ le système fondamental de voisinages de l'origine défini par

$$
V_{n \varepsilon}=\left\{f \in A ;\left\|\left.f\right|_{\hat{K}_{n}}\right\| \leqslant \varepsilon\right\} .
$$

D'après la Proposition 1 le cône $C$ s'identifie à un sous-cône dense dans $A_{+}^{\prime}$. Montrons l'égalité $C=A_{+}^{\prime}$. Pour tout entier $n$ le polaire de $V_{n, s}$ est:

$$
V_{n, \varepsilon}^{\circ}=1 / \varepsilon c\left(\hat{K}_{n} \cup-\hat{K}_{n}\right) .
$$

et par conséquent

$$
V_{n, \varepsilon}^{\circ} \cap C=\hat{K}_{n}
$$

D'après le théorème de Krein-Šsmulian, le cône $C$ est donc $\sigma(C, A)$ fermé et par suite coincide avec $A_{+}^{\prime}$. Pour montrer que $C$ est $\sigma(C$, $A$ )-complet nous établissons d'abord que $A$ est positivement engendré. Soit $V$ un voisinage de 0 dans $A$; si $V^{+}$est la partie positive de $V$ et $V^{\circ}$ son polaire, les égalités suivantes sont vérifiées:

(a) $V^{+}=\left(V^{\circ}+C\right)^{\circ}$

(b) $-V^{+}=\left(V^{\circ}-C\right)^{\circ}$

En effet, il suffit d'établir la première égalité. Soient $f$ dans $V^{+}$ et $x$ dans $V^{\circ}$ alors $f(x) \geqslant-1$. Pour tout $y \in C$ on a $f(y) \geqslant 0$, par suite $f(x+y) \geqslant-1$; ce qui entraine $V^{+} \subset\left(V^{\circ}+C\right)^{\circ}$. Inversement, si $f$ est une fonction de $\left(V^{\circ}+C\right)^{\circ}$, pour tout $x$ dans $V^{\circ}$ et $y$ dans $C$, on a: 


$$
f(x) \geqslant-1 \text { et } f(y) \geqslant 0 .
$$

Par suite $\left(V^{\circ}+C\right)^{\circ} \subset V^{+}$; d'où l'égalité (a).

Par conséquent, les ensembles $\left(V^{\circ}+C\right)$ et $\left(V^{\circ}-C\right)$ étant fermés dans $A^{\prime}$, on a:

$$
\left[c\left(V^{+} U-V^{+}\right)\right]^{\circ}=\left(V^{\circ}+C\right) \cap\left(V^{\circ}-C\right) .
$$

Comme il existe un système fondamental de voisinages de 0 de la forme $V_{n, \varepsilon}=\varepsilon V_{n 1}$, dans ce qui suit il suffit de considérer les voisinages $V_{n 1}$. Le théorème des bipolaires entraine

$$
V_{n, 1}^{\circ}=c\left(\hat{K}_{n} \subset-\hat{K}_{n}\right)
$$

par suite:

$$
\left(V_{n, 1}^{\circ}+C\right) \cap\left(V_{n, 1}^{\circ}-C\right)=(-\hat{K}+C) \cap\left(\hat{K}_{n}-C\right) .
$$

En effet, soit $1=y+p=\lambda y_{1}-(1-\lambda) y_{2}+p$; où $y_{1} \in \hat{K}_{n}, p \in C$,

$$
1=x-q=\lambda^{\prime} x_{1}-\left(1-\lambda^{\prime}\right) x_{2}-q \text {; où } x_{i} \in \widehat{K}_{n}, q \in C ;
$$

par conséquent: $-(1-\lambda) y_{2} \leqslant 1 \leqslant \lambda^{\prime} x_{1}$.

Ceci veut dire $1 \in\left(-\widehat{K}_{n}+C\right) \cap\left(\hat{K}_{n}-C\right)$, d'où l'inclusion:

$$
\left(V_{n, 1}^{\circ}+C\right) \cap\left(V_{n, 1}^{\circ}-C\right) \subset\left(-\hat{K}_{n}+C\right) \cap\left(\hat{K}_{n}-C\right)
$$

L'inclusion inverse est triviale.

Or $\left(-\hat{K}_{n}+C\right) \cap\left(\hat{K}_{n}-C\right) \subset 4 c\left(\hat{K}_{n} \cup-\hat{K}_{n}\right)$. En effet, si 1 est un point de $\left(-\hat{K}_{n}+C\right) \cap\left(\hat{K}_{n}-C\right)$, ils existent $x$ et $y$ dans $\hat{K}_{n}$ tels que $-x \leqq 1 \leqq y$. Par conséquent $0 \leqslant 1+x \leqslant y+x \in 2 \hat{K}_{n}$ Par suite, $1+x \in 2 \hat{K}_{n}$ et:

$$
\left(-\hat{K}_{n}+C\right) \cap\left(\hat{K}_{n}-C\right) \subset 2\left(\hat{K}_{n}-\hat{K}_{n}\right) .
$$

Mais par ailleurs on a toujours $\hat{K}_{n}-\hat{K}_{n} \subset 2 c\left(\hat{K}_{n} \cup-\hat{K}_{n}\right)$. Par conséquent $\left[c\left(V_{n, 1}^{+} U-V_{n, 1}^{+}\right)\right]^{\circ} \subset 4 V_{n, 1}^{\circ}$; et d'après le théorème des bipolaires:

$$
\left.V_{n, 1} \subset 4 \overline{c\left(V_{n, 1}^{+} U-V_{n, 1}^{+}\right.}\right) \subset 4\left(\overline{\left.V_{n, 1}^{+}-V_{n, 1}^{+}\right)} .\right.
$$

L'espace $H=A_{+}-A_{+}$muni de la topologie dont un système fondamental de voisinages de 0 est formé par les ensembles $V^{+}-V^{+}$est un espace de Frechet (Lemme 3) et l'application identique de $H$ dans $A$ est continue et presque-ouverte; elle est donc ouverte d'après le théorème des homomorphismes de Banach. Par conséquent:

$$
V_{n_{1}} \subset 4 \lambda\left(V_{n, 1}^{+}-V_{n, 1}^{+}\right) \text {pour tout } \lambda>1 \text {. }
$$

En particulier l'espace $A$ est positivement engendré. Soit $\mathscr{F}$ un filtre de Cauchy sur $C$ pour la structure uniforme $\sigma(C, A)$, l'application $p$ de $A$ dans $R$ définie par $p(f)=\lim _{\mathscr{F}} f$ est une forme linéaire positive 
sur $A$. D'après le Corollaire 4 cette application est continue par conséquent c'est un point de $C$. Or le filtre $\mathscr{F}$ est $\sigma(C, A)$-convergent vers $p$; par suite, le cône $C$ est $\sigma(C, A)$-complet.

Définition 6. Un cône convexe est $D-F$-bien coiffé s'il est recouvert par une famille $\left(K_{n}\right)_{n \in N}$ dénombrable et filtrante croissante de chapeaux.

Corollaire 7. Si le cône $C$ est $D-F$-bien coiffé, l'espace $A$ est positivement engendré et $C$ est complet pour la structure uniforme $\sigma(C, A)$.

La démonstration est une conséquence du Théorème 5 et de la remarque qui le précède.

Supposons le cône $C$ engendré par un convexe compact $K$ et notons $p$ sa jauge. Nous dirons que $p$ est $\alpha$-croissante si $x \leqslant y$ implique $p(x) \leqslant \alpha p(y)$ et qu'elle est $\alpha$-additive si $\alpha p(x+y) \geqslant p(x)+p(y)$ pour tout couple de points $x$ et $y$ dans $C$.

Proporition 8. Si le cône $C$ est engendré par un convexe compact $K$, les assertions suivantes sont équivalentes:

(a) $C$ est $(C, A)$-complet.

(b) $\hat{K}$ est $(C, A)$-compact.

(c) $B=c(K \cup-K) \cap C$ est $\sigma\left(C, E^{\prime}\right)$-compact et absorbe $\hat{K}$.

(d) $B$ est $\sigma\left(C, E^{\prime}\right)$-compact et sa jauge est $\alpha$-croissante pour un $\alpha>0$.

(e) $B$ est $\sigma\left(C, E^{\prime}\right)$-compact et sa jauge est $\alpha$-additive pour un $\alpha>0$.

DÉmonstration. (a) $\Rightarrow$ (b) est une conséquence du Théorème 5 (b) $\Rightarrow$ (c) L'ensemble $B$ est l'intersection de la boule unité de $A^{\prime}$ avec $C$. Il est clair que $B \subset \hat{K}$; par suite $B=c(K \cup-K) \cap \hat{K}$ est compact. D'après le théorème de Krein-Š́mulian le cône $C$ est $\sigma(C, A)$-fermé, par conséquent il coincide avec $A_{+}^{\prime}$. Le convexe $\hat{K}$ étant $\sigma(C, A)$ compact il est borné pour la topologie forte de $A^{\prime}$ et absorbé par $B$. Les assertions $(\mathrm{c}) \Rightarrow(\mathrm{d}) \Rightarrow(\mathrm{e})$ sont simples à démontrer en remarquant que $\hat{B}=\hat{K}$. L'assertion (e) $\Rightarrow$ (a) est une conséquence d'un résultat d'Asimow [2].

Dans la suite de cette section, nous supposerons que le cône $C$ est recouvert par une famille dénombrable et filtrante croissante de convexes compacts et qu'il est $\sigma(C, A)$-complet.

Corollaire 9. Si $B$ est un ensemble $\sigma(C, A)$-borné, il existe un entier $n_{\circ}$ tel que $\hat{K}_{n}$ absorbe $B$. Par suite les structures uniformes 
induites sur $B$ par $\sigma\left(C, E^{\prime}\right)$ et $\sigma(C, A)$ sont identiques.

DÉmonstration. Le convexe $[c(B \cup-B)]^{\circ}$ est un tonneau dans $A$; ce dernier espace est tonnelé car il est mètrique complet. Il existe donc $n_{\circ} \in N$ et $\varepsilon>0$ tels que $V_{n, \varepsilon} \subset[c(B-B)]^{\circ}$. En prenant les polaires des deux membres:

$$
V_{n, 1}^{\circ} \supset \varepsilon[c(B \cup-B)]^{\circ}=\overline{\varepsilon c(B \cup-B)} \supset \varepsilon B .
$$

Comme $V_{n, 1}^{\circ} \cap C=\hat{K}_{n}$, il s'en suit que $\varepsilon B \subset \hat{K}_{n}$. La dernière assertion est une conséquence de ce qui précède.

Corollaire 10. Si $K$ est un ensemble convexe $\sigma\left(C, E^{\prime}\right)$-compact, $i l$ existe un entier $n_{\text {。 }}$ tel que $\hat{K}_{n}$ absorbe $K$.

Démonstration. L'espace $A$ étant positivement engendré toute fonction de $A$ est majorée par une fonction de $A_{+}$, d'autre part une fonction affine positive est bornée sur un convexe compact. Ceci prouve que $K$ est $\sigma(C, A)$-borné et on est ramené au corollaire précédent.

Corollaire 11. L'espace $A$ ne dépend pas de la famille $\left(K_{n}\right)_{n \in N}$ choisie. Il s'identifie à l'espace des formes linéaires sur $E$ dont la restriction à tout convexe $\sigma\left(C, E^{\prime}\right)$-compact est continue.

La démonstration est une conséquence directe de la propriété d'absorption du Corollaire 10.

Corollaire 12. Si le cône $C$ est $D-F$-bien coiffé tout convexe $\sigma\left(C, E^{\prime}\right)$-compact est absorbé par un chapeau de la famille $\left(K_{n}\right)_{n \in N}$; de plus la famille des chapeaux de $C$ est filtrante croissante.

Démonstation. La première partie est une conséquence du Corollaire 10. Si $K^{\prime}$ et $K^{\prime \prime}$ sont deux chapeaux de $C$, l'ensemble $K=$ $c\left(K^{\prime} \cup K^{\prime \prime}\right)$ est un convexe $\sigma\left(C, E^{\prime}\right)$-compact; par suite, il est absorbé par un chapeau de la famille $\left(K_{n}\right)_{n \in N}$.

Corollaire 13. Soit $C$ un cône engendré par un convexe compact $K$ dont la jauge $p$ est $\alpha$-additive; $K$ absorbe tout convexe $\sigma\left(C, E^{\prime}\right)$ compact.

Démonstration. Le cône $C$ est $\sigma(C, A)$-complet d'après le Théorème 8 , de plus $K$ absorbe $B$ et par conséquent $\hat{K}$. On conclut grace au Corollaire 10. 
EXEMPLE 14. Le résultat du Corollaire 9 est en défaut si $K$ n'est plus supposé convexe; plus précisément:

Il existe un cône $C$ à chapeau universel et une suite $\left(x^{m}\right)_{m \in N}$ convergente pour $\sigma\left(C, E^{\prime}\right)$ mais non $\sigma(C, A)$-bornée. En effet, soit $C=l_{+}^{1}(N)$ le cône des suites sommables à termes positifs; si $E^{\prime}$ est l'espace $\boldsymbol{R}^{(N)}$, la topologie $\sigma\left(C, E^{\prime}\right)$ est la trace de la topologie produit sur $l_{+}^{1}(N)$. Le cône $l_{+}^{1}(N)$ admet pour chapeau universel $K_{\circ}=$ $\left\{\left(x_{n}\right)_{n \in N} ; \Sigma x_{n} \leqslant 1\right\}$, et l'espace $A$ s'identifie à l'espace $\mathscr{C}_{\circ}(N)$ des suites tendant vers 0 . Soit $\left(x^{m}\right)_{m \in N}$ la suite de $l_{+}^{1}(N)$ définie par:

$$
x_{n}^{m}=m \delta_{m n} \text { (où } \delta_{m n} \text { est le symbole de Kronecker). }
$$

La suite $\left(x^{m}\right)_{m \in N}$ est $\sigma\left(C, E^{\prime}\right)$-convergente vers 0 mais n'est pas $\sigma(C$, A)-bornée, sinon elle serait fortement bornée or $\sum_{n \in N} x_{n}^{m}=m$ n'est pas bornée indépendemment de $m$.

L'exemple précédent montre en particulier que le plongement de $C$ sur $A_{+}^{\prime}$ n'est pas une homéomorphie.

EXEMPLe 15. Si la famille des convexes compacts recouvrant $C$ n'est pas supposée dénombrable, la conclusion du Théorème 5 ne subsiste plus:

Il existe un cône $C$ recouvert par une famille filtrante croissante de chapeaux et tel que la structure uniforme $\sigma(C, A)$ ne soit pas complète:

Soit $\Omega$ le plus petit ordinal non dénombrable et $X=[0, \Omega[$ muni de la topologie de l'ordre. On sait que $X$ est un espace localement compact. Le cône $C$ des mesures positives à support compact sur $X$ est recouvert par une famille (non dénombrable) filtrante croissante de chapeaux $\left(K_{\alpha, n}\right)$ à savoir:

$$
K_{\alpha, n}=\{\mu \in C ; \text { Support }(\mu) \subset[0, \alpha] ;\|\mu\| \leqslant n ; \alpha \in X\} .
$$

L'espace $\mathscr{C}(X)$ des fonctions continues sur $X$ s'identifie trivialement à un sous-espace de $A$. Inversement, soient $f$ une fonction de $A$ et $\alpha$ un point de $X$; la fonction $f$ est continue sur tout chapeau $K_{\alpha, n}$, par suite elle est continue sur les points extrêmaux de $K_{\alpha, n}$; la fonction $f$ est donc le prolongement à $C$ d'une fonction de $\mathscr{C}(X)$. De plus, l'espace $M_{K}=(C-C)$ des mesures à support compact est le dual de $\mathscr{C}(X)$ muni de la topologie de la convargence uniforme sur tout compact. Soit $\mathscr{F}=\left(S_{\alpha}\right)_{\alpha \in X}$ l'ensemble des parties ne $C$ de la forme $S_{\alpha}=\left\{\varepsilon_{x} ; x \geqq \alpha ; \alpha \in X\right\} ; \mathscr{F}$ constitue une base de filtre de Cauchy pour $\sigma(C, \mathscr{C}(X))$, en effet toute fonction de $\mathscr{C}(X)$ est constante à partir d'un certain rang, par suite il existe $\alpha_{f} \in X$ tel que pour tous $x$ et $y$ dans $S_{\alpha_{f}}$ on ait $|f(x)-f(y)|=0$. 
Pourtant la base de filtre $\mathscr{F}$ ne converge pas dans $C$ car la forme linéaire $p$ dèfinie par $p(f)=\lim _{\mathscr{J}} f=\lim _{x \rightarrow \Omega} f(x)$ n'est pas continue sur $\mathscr{C}(X)$. En effet si $\alpha \in X$ la fonction:

$$
\begin{array}{ll}
f_{\alpha}=1 & \text { sur }[0, \alpha] ; \\
f_{\alpha}=0 & \text { sur }[\alpha+1, \Omega[
\end{array}
$$

est continue et la famille $\left(f_{\alpha}\right)_{\alpha \in X}$ converge uniformément sur tout compact vers la fonction $f=1$. Mais il est clair que pour tout $\alpha \epsilon$ $X$ on $a p\left(f_{\alpha}\right)=0$ et pourtant $p(f)=1$. La structure uniforme $\sigma(C$, $\mathscr{C}(X))$ n'est donc pas complète.

Remarquons que dans ce cas particulier $C$ est identique au cône des mesures positives bornées sur $X$ et il est complet pour la dualité $\sigma\left(C, \mathscr{E}_{\circ}(X)\right)$. Cet exemple montre aussi que si la famille des chapeaux n'est pas dénombrable les conclusions des Corollaires 9 et 11 sont en défaut.

Exemple 16. Si $C$ est le cône $\boldsymbol{R}_{+}^{(I)}$ on sait d'après un résultat de G. Choquet [4] que $\sigma\left(\boldsymbol{R}_{+}^{(I)}, \boldsymbol{R}^{I}\right)$ n'est complète que si $I$ a un cardinal modéré. On ignore s'il existe une structure uniforme faible complète sur le cône $\boldsymbol{R}_{+}^{(I)}$ pour un cardinal $I$ quelconque.

III. Structures uniformes sur un convexe bien coiffé. Soit $X$ un convexe d'un e.l.c. $E$, un sous-convexe compact $K$ de $X$ est dit un chapeau si son complémentaire dans $X$ est un convexe. Un tel chapeau est universel si la variété affine engendrée par $K$ contient $X$. On étend de façon naturelle les notions de bien coiffé et de $D-$ $F$-bien coiffé.

Désignons par $\alpha(X)$ le cône asymptote de $X$; c'est-à-dire:

$$
\left.\alpha(X)=\bigcap_{\lambda>0} \lambda(X-x) \text { (où } x \in X\right) ;
$$

et par $P(X)$ le cône de l'espace $F=E \times R$ défini par:

$P(X)=\{(x, r) ; r>0$ et $x / r \in X$; ou bien $r=0$ et $x \in \alpha(X)\}$ Si $X$ est fermé dans $E$, le cône $\alpha(X)$ est indépendant du point $x$ choisi, et on peut aisément vérifier que $\alpha(X)$ et $P(X)$ sont fermés. Le lemme suivant est dû à Asimow [1]:

Lemme 17. Soit $X$ un convexe fermé dans un e.l.c. $E$, pour que $P(X)$ soit bien coiffé (resp. $D-F$-bien coiffé; resp. à chapeau universel) il faut et il suffit que $X$ vérifie la même propriété. De plus tout chapeau de $X$ est la trace sur $X$ d'un chapeau de $P(X)$.

THÉonème 18. Soit $X$ un convexe fermé; si $X$ est $D-F$-bien coiffé il est complet pour la plus fine des structures uniformes faibles 
compatibles avec la topologie des sous-convexes compacts de $X$.

Démonstration. Le cône $P(X)$ étant $D-F$-bien coiffé il est complet pour la structure uniforme $\sigma(P(X), A)$ d'après le Corollaire 7. Le convexe $X$ s'identifie à un fermé de $F$ muni de la topologie produit, à fortiori il est fermé pour $\sigma(P(X), A)$. La stucture uniforme induite sur $X$ par $\sigma(P(X), A)$ est la plus fine des structures uniformes faibles compatibles avec la topologie des chapeaux de $X$. Le théorème se déduit alors du Corollaire 10 et du Lemme 17.

Ceci nous permet de retrouver le résultat connu suivant:

Corollaire 19. Si $X$ est un convexe fermé localement compact saillant il est complet pour la plus fine des strutures uniformes compatibles avec la topologie des convexes compacts de $X$.

DÉmonstration. D'après un résultat connu de Klee tout convexe fermé localement compact saillant admet un chapeau universel; on conclut grâce au théorème précédent.

ExEMPLe 20. La conclusion du Théorème 18 est en défaut si on ne suppose plus que $X$ est fermé.

Soit $X$ le convexe de $\boldsymbol{R}^{2}$ défini par:

$$
X=\{(x, y) ; 0 \leqslant x<1 \text { et } y \geqslant 0 \text {, ou bien } x=1 \text { et } y=0\} .
$$

Ce convexe est $D-F$-bien coiffé; en effet, soit $\left(x_{\circ}, y_{\circ}\right)$ un point de $X$, la droite joignant ce point à $(1,0)$ rencontre l'axe $\{x=0, y \geqslant 0\}$ et la partie de $X$ qui est en dessous de cette droite est un chapeau de $X$ contenant $\left(x_{\circ}, y_{\circ}\right)$. Il est clair que $X$ n'étant pas fermé ne peut pas être complet dans $\boldsymbol{R}^{2}$.

IV. Comparaison des structures uniformes faibles sur C. Supposons que le cône $C$ soit recouvert par une famille $\left(K_{n}\right)_{n \in N}$ dénombrable et filtrante croissante de convexes compacts tels que la struture uniforme $\sigma(C, A)$ soit complète.

Les deu structures uniformes $\sigma\left(C, E^{\prime}\right)$ et $\sigma\left(C, E^{\prime}\right)$ et $\sigma(C, A)$ sont en général distinctes, en fait:

Proposition 21. Pour que les structures $\sigma\left(C, E^{\prime}\right)$ et $\sigma(C, A)$ soient égales $i l$ faut et il suffit que $E^{\prime}$ et $A$ coincident.

DÉmonstration. La condition est suffisante. Inversement, soit $f \in A$, il existe $\left(l_{i}\right)_{i=1, \ldots, n}$ appartenant à $E^{\prime}$ tels que: 
$\bigcap_{1}^{h}\left\{(x, y) \in C_{x} C ;\left|l_{i}(x-y)\right| \leqslant 1\right\} \subset\left\{(x, y) \in C_{x} C ;|f(x-y)| \leqslant 1\right\}$.

Par suite si $z \in E$ est un point tel que $\left|l_{i}(z)\right| \leqslant 1 i=1, \cdots, n$, il s'écrit $z=x-y$ avec $(x, y) \in C_{x} C$ tel que $\left|l_{i}(x-y)\right| \leqslant 1 i=1, \cdots, n$. Par conséquent $|f(z)| \leqslant 1$; ce qui veut dire $\sigma\left(E, E^{\prime}\right)=\sigma(E, A)$, d'où l'égalité $E^{\prime}=A$.

Proposition 22. Si les filtres de voisinages de 0 dans $C$ pour les topologie induites par $\sigma\left(C, E^{\prime}\right)$ et $\sigma(C, A)$ sont identiques, la structure uniforme $\sigma\left(C, E^{\prime}\right)$ est quasi-complète.

Démonstration. Soit $B$ une partie de $C$ bornée et fermée pour $\sigma\left(C, E^{\prime}\right)$, à fortiori $B$ est $\sigma(C, A)$-fermée donc $\sigma(C, A)$-complète. La partie $B$ étant bornée, elle est absorbée par tout voisinage de 0 pour les topologies induites par $\sigma\left(C, E^{\prime}\right)$ et $\sigma(C, A)$; elle est donc $\sigma(C, A)$ bornée. Il existe un entier $n_{\circ}$ tel que $\hat{K}_{n}$ o absorbe $B$ (Corollaire 9) et les structures uniformes induites sur $B$ coincident. En particulier $B$ est $\sigma\left(C, E^{\prime}\right)$-complète.

TÉoRÈme 23. Si la structure uniforme $\sigma\left(C, E^{\prime}\right)$ est quasi-complète les ensembles bornés sont les mêmes pour $\sigma\left(C, E^{\prime}\right)$ et $\sigma(C, A)$. De plus, les structures uniformes induites sur les bornés sont identiques.

Démonstration. Soit $B \subset C$ une partie $\sigma\left(C, E^{\prime}\right)$-bornée, l'adhérence dans $C$ de $c(B)$ est un convexe saillant faiblement complet; il existe un ensemble $I$ tel que $\overline{c(B)}$ se plonge dans un borné de $\boldsymbol{R}_{+}^{I}$ (3). Il s'en suit que $\overline{c(B)}$ est compact et par suite il existe un $\hat{K}_{n}$ qui absorbe $\overline{c(B)}$ (Corollaire 10). Les structures uniformes induites $\operatorname{sur} B$ sont identiques.

Corollaire 24. Supposons que la structure uniforme $\sigma\left(C, E^{\prime}\right)$ soit quasi-complète et 'soit $\mathscr{F}$ un filtre $\sigma\left(C, E^{\prime}\right)$-convergent. Si $\mathscr{F}$ vérifie l'une des deux conditions suivantes il est $\sigma(C, A)$-convergent:

(a) F्F est porté par un ensemble borné,

(b) $\mathscr{F}$ est à base dénombrable.

DÉmonstration. (a) est une conséquence du Théorème 23. (b). Soit $x$ la limite de $\mathscr{F}$ pour la topologie induite par $\sigma\left(C, E^{\prime}\right)$. Si $\mathscr{F}$ est le filtre élémentaire associé à suite $\left(x_{n}\right)_{n \in N}$, l'ensemble $K=\left(x_{n}\right)_{n \in N}$ $x$ est $\sigma\left(C, E^{\prime}\right)$-compact. La structure uniforme $\sigma\left(C, E^{\prime}\right)$ étant quasicomplète, les topologies induites sur $K$ coincident, et la suite $\left(x_{n}\right)_{n \in N}$ converge vers $x$ pour $\sigma(C, A)$. Supposons que $\mathscr{F}$ soit un filtre à base dénombrable quelconque; tout filtre élémentaire plus fin converge vers $x$ pour $\sigma\left(C, E^{\prime}\right)$ donc aussi pour $\sigma(C, A)$. Il en est par conséquent de 
même pour $\mathscr{F}$ puisqu'il est l'intersection des filtres élémentaires plus fin que lui.

Corollaire 25. Supposons $\sigma\left(C, E^{\prime}\right)$ quasi-complète; pour tout sousespace $F$ de $A$ qui contient $E^{\prime}$ la structure uniforme $\sigma(C, F)$ est quasi-complète.

Démonstration. Il suffit de montrer que les filtres de Cauchy portés par les ensembles bornés de $C$ sont les mêmes pour les structures uniformes $\sigma\left(C, E^{\prime}\right)$ et $\sigma(C, A)$. Or ceci est une conséquence du Corollaire 24.

DÉfinition 26. L'espace $E^{\prime}$ est dominant dans $A$ si toute fonction de $A_{+}$est majorée fur $C$ par une fonction de $E_{+}^{\prime}$.

TÉoRÈme 27. Supposons que le cône $C$ est $\sigma(C, A)$-complet; si l'espace $E^{\prime}$ est positivement engendré, les assertions suivantes sont équivalentes:

(a) L'espace $E^{\prime}$ est dominant dans $A$.

(b) Les topologies induites par $\sigma\left(C, E^{\prime}\right)$ et $\sigma(C, A)$ sont identiques.

(c) Le point 0 admet le même filtre de voisinages dans $C$ pour les topologies induites par $\sigma\left(C, E^{\prime}\right)$ et $\sigma(C, A)$.

De plus si l'une des conditions précédentes est vérifiée elle implique:

(d) Le structure uniforme $\sigma\left(C, E^{\prime}\right)$ est complète.

DÉmonstration. (a) $\Rightarrow(b)$. Soit $\mathscr{U}$ un ultafiltre $\sigma\left(C, E^{\prime}\right)$-convergent et $f$ une fonction de $A$; il existe une fonction 1 de $E_{+}^{\prime}$ qui majore $|f|$ sur $C$. La base d'ultrafiltre $f(\mathscr{C})$ est donc portée par un ensemble borné de $\boldsymbol{R}$, et par suite est convergente. Le filtre $\mathscr{C}$ est par conséquent un filtre de Cauchy pour $\sigma(C, A)$. Le cône $C$ étant $C$ complet pour $\sigma(C, A)$, le filtre $\mathscr{C}$ est $\sigma(C, A)$-convergent. (b) implique trivialement (c). (c) $\Rightarrow$ (a). Soit $f$ une fonction de $A_{+}$, il existe $\left(l_{i}\right)_{i=1, \ldots, n}$ appartenant à $E^{\prime}$ telles que:

$$
\bigcup_{1}^{n}\left\{x \in C ;\left|l_{i}(x)\right| \leqslant 1\right\} \subset\{x \in C ; f(x) \leqslant 1\} .
$$

L'espace $E^{\prime}$ étant positivement engendré, pour tout $i=1, \cdots, n$ il existe $l_{i}^{\prime}$ et $l_{i}^{\prime \prime}$ dans $E_{+}^{\prime}$ telles que $l_{i}=l_{i}^{\prime}-l_{i}^{\prime \prime}$; par suite:

$$
\bigcap_{1}^{h}\left\{x \in C ;\left(l_{i}^{\prime}+l_{i}^{\prime \prime}\right)(x) \leqslant 1\right\} \subset\{x \in C ; f(x) \leqslant 1\} .
$$

Par homogénéité on conclut que $f \leqslant \sum_{1}^{h}\left(l_{1}^{\prime}+l_{i}^{\prime \prime}\right)$; ce qui établit (a). $(a) \Rightarrow(d)$. Soit $\mathscr{F}$ un filtre de Cauchy pour la structure uniforme 
$\sigma\left(C, E^{\prime}\right)$; tout ultrafiltre plus fin est de Cauchy; par suite on peut montrer qu'il est $\sigma(C, A)$-convergent comme dans $(\mathrm{a}) \Rightarrow(\mathrm{b})$. A fortiori le filtre $\mathscr{F}$ est $\sigma\left(C, E^{\prime}\right)$-convergent.

L'assertion (d) n'implique pas (a); en effet, la proposition suivante dûe à G. Choquet fournit un contre-exemple:

Proposition 28. Il existe sur le cône $l_{+}^{1}(N)$ une infinité de structures uniformes faiblement complètes compatibles avec la topologie du chapeau, mais induisent des topologies différentes sur $l_{+}^{1}(N)$.

DÉmonstration. Soient $f_{0}$ une fonction strictement positive de $\mathscr{C}_{0}(N)$ et $\mathscr{Q}$ un ultrafiltre non trivial sur $N$; on note:

$$
W\left(f_{0}, \mathscr{U}\right)=\left\{g \in \mathscr{C}_{0}(N) ; \lim _{\mathscr{U}} g / f_{0}=0\right\} .
$$

Cet espace contient $\boldsymbol{R}^{(N)}$, par suite il sépare les points de $l_{+}^{1}(\boldsymbol{N})$. La topologie induite sur $l_{+}^{1}(N)$ par la dualité avec $W\left(f_{0}, \mathscr{Q} \ell\right)$ est donc compatible avec la topologie $d u$ chapeau. Il est clair d'autre part que l'espace $W\left(f_{0}, \mathscr{C}\right)$ n'est pas dominant dans $\mathscr{C}_{0}(\boldsymbol{N})$; en effet la fonction $f_{0}$ n'est majorée par aucune fonction positive de $W\left(f_{0}, \mathscr{U}\right)$. Par suite les topologies induites sur $l_{+}^{1}(N)$ par les dualités avec $W\left(f_{0}, \mathscr{Q}_{6}\right)$ et $\mathscr{C}_{0}(\boldsymbol{N})$ sont différentes. Pour montrer que la structure uniforme $\sigma\left(l_{+}^{1}(N), W\left(f_{0}, \mathscr{U}\right)\right)$ est complète il suffit de montrer que toute forme linéaire positive sur $W\left(f_{0}, \mathscr{Q}\right)$ est une mesure bornée sur $N$; c'est-à-dire un point de $l_{+}^{1}(N)$. Soit 1 une telle forme linéaire, pour tout $n \in N$ on pose $a_{n}=1\left(\delta_{m n}\right)$ il s'agit de montrer que pour tout $g \in$ $W(0, \mathscr{Q})$ on a:

$$
l(g)=\sum_{n \in N} a_{n} g(n) .
$$

La série de terme général $\left(a_{n}\right)_{n \in N}$ est sommable; sinon il existe une partie $P \subset N$ telle que $\sum_{n \in P} a_{n}=+\infty$ et $\sum_{n \in N / P} a_{n}=+\infty$. Supposons $P \notin \mathscr{U}$ et $f \in W\left(f_{0}, \mathscr{U}\right)$, la fonction $f$ restriente à $P$ est arbitraire dans $\mathscr{C}_{0}(P)$. Par suite $\sum_{n \in P} a_{n} f(n) \leqq 1(f)<+\infty$ entraine que $\left(a_{n}\right)_{n \in P} \in l_{+}^{1}(P)$ ce qui contredit $\sum_{n \in P} a_{n}=+\infty$. Posons pour tout $g \in W\left(f_{0}, \mathscr{C}\right): \mathrm{l}^{\prime}(g)=\mathrm{l}(g)-\sum_{n \in N} a_{n} g(n)$; c'est une forme linéaire positive sur $W\left(f_{0}, \mathscr{Q}\right)$, nulle sur $\boldsymbol{R}^{(N)}$. On peut vérifier que $\mathrm{l}^{\prime}(f)=0$ pour tout $f \in W\left(f_{0}, \mathscr{W}\right)$ tel qu'il existe $B \in \mathscr{C}$ pour lequel $\left.f\right|_{B}=0$. Ceci étant fait, on remarque que pour tout $f \geqslant 0 W\left(f_{0}, \mathscr{U}\right)$ il existe une fonction positive $g \in W\left(f_{0}, \mathscr{C}\right)$ infiniment plus grande que $f$ sur $\mathscr{C}$; d'où, si $l^{\prime}(f)=1$ on a $l^{\prime}(g)=\infty$, ce qui est absurde. Par conséquent 1 s'identifie à $\left(a_{n}\right)_{n \in N}$. Si $\mathscr{U}$ et $\mathscr{C}^{\prime}$ sont deux ultrafiltres non triviaux distincts, il est simple de vérifier que les topologies induites 
sur $l_{+}^{1}(\boldsymbol{N})$ par les dualités avec $W\left(f_{0}, \mathscr{C}\right)$ et $W\left(f_{0}, \mathscr{C}^{\prime}\right)$ sont différentes, ce qui établit la proposition.

\section{REFERENCES}

1. L. Asimow, Extreme structure of well-capped convex sets, Trans. Amer. Math. Soc., 138 (1969), 363-375.

2. - Directed Banach spaces of affine functions, Trans. Amer. Math. Soc., 143 (1969), 117-131.

3. G. Choquet, Ensembles et cônes convexes faiblement complets, C. R. Acad. Sci., Paris série A 254 (1962), 908-1910.

4. — Cardinaux 2-mesurables et cônes faiblement complets, Ann. Inst. Fourier (Grenoble), 17 (1967), 383-393.

5. - Caractère faiblement complet des cônes à chapeau universel, Bull. Sci. Math., 94 (1970), 281-288.

6. H. Fakhoury, Structures uniformes sur un cône bien coiffé, C. R. Acad. Sci, Paris, Série A 270 (1970), 1365-1368.

7. H. Schaefer, Topological Vector Spaces, Macmillan series in advanced mathematics and theoretical physics, (1966).

Received May 25, 1970 and in revised form August 17, 1970.

Département de mathématiques, Paris 


\title{
PACIFIC JOURNAL OF MATHEMATICS
}

\author{
EDITORS
}

\author{
H. SAMELSON \\ Stanford University \\ Stanford, California 94305 \\ C. R. HOBBY \\ University of Washington \\ Seattle, Washington 98105
}

J. DugundJI

Department of Mathematics

University of Southern California

Los Angeles, California 90007

RICHARD ARENS

University of California

Los Angeles, California 90024

\section{ASSOCIATE EDITORS}
E. F. BeCKenBaCH
B. H. NeUMANN
F. WOLF
K. Yoshida

\section{SUPPORTING INSTITUTIONS}

\author{
UNIVERSITY OF BRITISH COLUMBIA \\ CALIFORNIA INSTITUTE OF TECHNOLOGY \\ UNIVERSITY OF CALIFORNIA \\ MONTANA STATE UNIVERSITY \\ UNIVERSITY OF NEVADA \\ NEW MEXICO STATE UNIVERSITY \\ OREGON STATE UNIVERSITY \\ UNIVERSITY OF OREGON \\ OSAKA UNIVERSITY
}

\author{
UNIVERSITY OF SOUTHERN CALIFORNIA \\ STANFORD UNIVERSITY \\ UNIVERSITY OF TOKYO \\ UNIVERSITY OF UTAH \\ WASHINGTON STATE UNIVERSITY \\ UNIVERSITY OF WASHINGTON \\ AMERICAN MATHEMATICAL SOCIETY \\ NAVAL WEAPONS CENTER
}

The Supporting Institutions listed above contribute to the cost of publication of this Journal, but they are not owners or publishers and have no responsibility for its content or policies.

Mathematical papers intended for publication in the Pacific Journal of Mathematics should be in typed form or offset-reproduced, (not dittoed), double spaced with large margins. Underline Greek letters in red, German in green, and script in blue. The first paragraph or two must be capable of being used separately as a synopsis of the entire paper. The editorial "we" must not be used in the synopsis, and items of the bibliography should not be cited there unless absolutely necessary, in which case they must be identified by author and Journal, rather than by item number. Manuscripts, in duplicate if possible, may be sent to any one of the four editors. Please classify according to the scheme of Math. Rev. Index to Vol. 39. All other communications to the editors should be addressed to the managing editor, Richard Arens, University of California, Los Angeles, California, 90024.

50 reprints are provided free for each article; additional copies may be obtained at cost in multiples of 50 .

The Pacific Journal of Mathematics is published monthly. Effective with Volume 16 the price per volume (3 numbers) is $\$ 8.00$; single issues, $\$ 3.00$. Special price for current issues to individual faculty members of supporting institutions and to individual members of the American Mathematical Society: $\$ 4.00$ per volume; single issues $\$ 1.50$. Back numbers are available.

Subscriptions, orders for back numbers, and changes of address should be sent to Pacific Journal of Mathematics, 103 Highland Boulevard, Berkeley, California, 94708.

PUBLISHED BY PACIFIC JOURNAL OF MATHEMATICS, A NON-PROFIT CORPORATION

Printed at Kokusai Bunken Insatsusha (International Academic Printing Co., Ltd.), 270, 3chome Totsuka-cho, Shinjuku-ku, Tokyo 160, Japan. 


\section{Pacific Journal of Mathematics}

\section{Vol. 39, No. $3 \quad$ July, 1971}

William O'Bannon Alltop, 5-designs in affine spaces ................... 547

B. G. Basmaji, Real-valued characters of metacyclic groups ................. 553

Miroslav Benda, On saturated reduced products....................... 557

J. T. Borrego, Haskell Cohen and Esmond Ernest Devun, Uniquely representable semigroups. II.......................................

George Lee Cain Jr. and Mohammed Zuhair Zaki Nashed, Fixed points and stability for a sum of two operators in locally convex spaces ....................

Donald Richard Chalice, Restrictions of Banach function spaces ...............

Eugene Frank Cornelius, Jr., A generalization of separable groups ..............

Joel L. Cunningham, Primes in products of rings ......................

Robert Alan Morris, On the Brauer group of $Z$.

593

603

615

David Earl Dobbs, Amitsur cohomology of algebraic number rings ...............

Charles F. Dunkl and Donald Edward Ramirez, Fourier-Stieltjes transforms and

weakly almost periodic functionals for compact groups ...................

Hicham Fakhoury, Structures uniformes faibles sur une classe de cônes et

d'ensembles convexes ......................................

Leslie R. Fletcher, A note on C $\theta \theta$-groups.

Humphrey Sek-Ching Fong and Louis Sucheston, On the ratio ergodic theorem for

semi-groups............................................

James Arthur Gerhard, Subdirectly irreducible idempotent semigroups ...........

Thomas Eric Hall, Orthodox semigroups.....................

Marcel Herzog, $C \theta \theta$-groups involving no Suzuki groups ..........

669

687

John Walter Hinrichsen, Concerning web-like continua ..........

691

Frank Norris Huggins, A generalization of a theorem of F. Riesz.

695

Carlos Johnson, Jr., On certain poset and semilattice homomorphisms

703

Alan Leslie Lambert, Strictly cyclic operator algebras ...........

717

Howard Wilson Lambert, Planar surfaces in knot manifolds . . .

727

Robert Allen McCoy, Groups of homeomorphisms of normed linear spaces ....... 735

T. S. Nanjundiah, Refinements of Wallis's estimate and their generalizations ...... 745

Roger David Nussbaum, A geometric approach to the fixed point index .......... 751

John Emanuel de Pillis, Convexity properties of a generalized numerical range .... 767

Donald C. Ramsey, Generating monomials for finite semigroups ....

783

William T. Reid, A disconjugacy criterion for higher order linear vector differential equations...

Roger Allen Wiegand, Modules over universal regular rings...

Kung-Wei Yang, Compact functors in categories of non-archimedean Banach

spaces.

R. Grant Woods, Correction to: "Co-absolutes of remainders of Stone-Čech compactifications".

Ronald Owen Fulp, Correction to: "Tensor and torsion products of

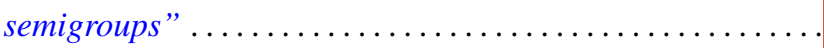

Bruce Alan Barnes, Correction to: "Banach algebras which are ideals in a banach 